\title{
Qualidade microbiológica e temperatura de dietas enterais antes e após implantação do sistema de análise de perigos e pontos críticos de controle
}

\author{
Microbiological quality and temperatures of enteral \\ feedings before and after implementation of \\ hazard analysis and critical control point
}

Miriam Isabel Souza dos Santos SIMON ${ }^{1}$

Susette FREIMÜLLER²

Eduardo César TONDO²

Anelise Siviero RIBEIRO'

Michele DREHMER ${ }^{1}$

RE S U M O

\section{Objetivos}

Avaliar a qualidade microbiológica e a temperatura de dietas enterais antes e após a implementação do sistema Análise de Perigos e Pontos Críticos de Controle na Central de Produção de Alimentação Enteral do Hospital de Clínicas de Porto Alegre.

\section{Métodos}

Durante o período de setembro de 2001 a janeiro de 2002, foram coletadas 320 amostras de 4 tipos de dietas enterais produzidas na Central de Produção de Alimentação Enteral (dietas padrão I, II, III e dieta especial), as quais foram submetidas a análises microbiológicas e de temperatura. As amostras foram coletadas semanalmente em dois pontos do processo: logo após o preparo e após 16 horas de armazenamento refrigerado. Para comparação dos dados pré e pós análise dos perigos em pontos criticos e de controle, utilizou-se o teste " $t$ " de Wilcoxon para amostras pareadas (teste não-paramétrico equivalente ao teste " $t$ " de Student para amostras pareadas) com nível de significância de 0,05.

\footnotetext{
1 Universidade Federal do Rio Grande do Sul, Hospital de Clínicas de Porto Alegre, Serviço de Nutrição e Dietética. R. Ramiro Barcelos, 2350, 90035-000, Porto Alegre, RS, Brasil. Correspondência para/Correspondence to: M.I.S.S. SIMON. E-mail: <misantos@hcpa.ufrgs.br>.

2 Universidade Federal do Rio Grande do Sul, Instituto de Ciência e Tecnologia dos Alimentos, Departamento de Ciências dos Alimentos. Porto Alegre, RS, Brasil.
} 
140 | M.I.S.S. SIMON et al.

\section{Resultados}

Antes da análise de perigos e pontos críticos de controle, 92\% das temperaturas de armazenamento estavam acima dos valores de refererência vigentes. Após a implantação da análise de perigos e pontos críticos de controle, houve reduções significantes $(p<0,05)$ nas contagens de microrganismos mesófilos totais das dietas II, III e Especial, logo após preparo. Reduções significantes também foram encontradas nas dietas II e III armazenadas sob refrigeração. Não foram encontrados coliformes fecais, Staphylococcus aureus, Bacillus cereus, Clostridium sulfito redutores, Salmonella sp., Yersinia enterocolitica ou Listeria monocytogenes em nenhum dos momentos avaliados. A análise crítica do fluxograma de produção identificou as etapas de aquisição de matéria-prima e de armazenamento sob refrigeração como Pontos Críticos de Controle das dietas produzidas.

\section{Conclusão}

Com a implantação deste sistema na central de produção de alimentação enteral foram realizadas mudanças que contribuíram significativamente na qualidade do produto final, diminuindo riscos de contaminação alimentar.

Termos de indexação: nutrição enteral; contaminação de alimentos; análise de perigos e pontos críticos de controle.

\section{A B S T R A C T}

\section{Objective}

This study evaluated microbiological quality and temperature of enteral formulas before and after the implementation of the Hazard Analysis Critical Control Points system at the Central of Enteral Feedings of Hospital de Clínicas de Porto Alegre.

\section{Methods}

During the period of September 2001 and January 2002, 320 samples of four kinds of enteral feeding identified as diets I, II, III, and Special diet were collected and submitted to temperature and microbiological analysis. Samples were collected weekly in two steps of the flow chart of production: immediately after preparation and after 16 hours of storage at refrigeration. For statistical analysis, the Wilcoxon's test was used.

\section{Results}

Before Hazard Analysis Critical Control Points system, 92\% of the storage temperatures were considered inadequate according to the Brazilian legislation. After Hazard Analysis Critical Control Points system implementation, significant reductions $(p<0.05)$ were observed in the counts of mesophilic microorganisms of diets II, III, and Special diet, immediately after preparation. Significant reductions were also observed in the diets II and III stored at refrigeration. Fecal coliforms, Staphylococcus aureus, Bacillus cereus, Clostridium sp., Salmonella sp., Yersinia enterocolitica or Listeria monocytogenes were not found in any of the analyzed samples. Hazard analysis identified the acquisition of raw materials and refrigerated storage as the Critical Control Points of the produced diets.

\section{Conclusion}

After the implementation of the Hazard Analysis Critical Control Points system at the Center of Enteral Feedings, the quality of the final product increased significantly, reducing the risk of food contamination.

Indexing terms: enteral nutrition; food contamination; hazard analysis critical control points system.

\section{N T R O D U Ç Ã O}

A nutrição enteral (NE), infundida por sonda, é um método seguro de prover nutrientes no qual a via digestiva fisiológica é utilizada para receber dieta. Ela é utilizada para fornecer suporte nutricional a indivíduos que não têm condições de se alimentar por via oral, mas que tenham um trato gastrintestinal funcionante ${ }^{1}$. O suporte nutricional é uma importante terapêutica para pacientes que sofreram cirurgia no trato gastrintestinal ou apresentam distúrbios gastrintestinais 
graves, lesões do sistema nervoso central, depressão, câncer, entre outros ${ }^{2}$. Atualmente, a NE é amplamente utilizada por ser segura, econômica e por preservar a imunidade e os mecanismos de defesa de pacientes, quando comparada com a nutrição parenteral ${ }^{3}$.

As dietas enterais são ricas em macro e micronutrientes, sendo, portanto, excelentes meios para crescimento de microorganismos ${ }^{4}$. A administração de dieta eventualmente contaminada por diferentes microorganismos pode causar distúrbios gastrintestinais como náuseas, vômitos ou diarréias, sendo esta última, a complicação mais comum em pacientes que recebem $\mathrm{NE}^{3}$. Além disso, contaminações de origem microbiana em NE já foram documentadas, causando severas complicações infecciosas como septicemia, bacteremia e pneumonia ${ }^{5}$. Sendo assim, a utilização de NE deve seguir cuidados e procedimentos criteriosos, principalmente com o objetivo de controlar as possíveis fontes de contaminação das formulações. Para tanto, a utilização do sistema de análise de perigos e pontos críticos de controle (APPCC) pode ser uma alternativa eficiente para garantir a qualidade microbiológica dessas formulações. Esse sistema permite identificar os perigos relacionados à produção de alimentos, possibilitando a fabricação de produtos isentos de contaminações biológicas, químicas e físicas.

A análise de perigos e pontos críticos de controle é um sistema contínuo e dinâmico, no qual as alterações e as medidas preventivas são aplicadas durante o processamento e não no produto final, quando já não restam alternativas além do descarte do produto 6 . Entre as ações necessárias à implantação do APPCC, pode-se destacar a análise dos perigos por meio de investigações das condições microbiológicas de matérias-primas, ambientes de preparação dos alimentos, utensílios e equipamentos, além de parâmetros de tempo e temperatura. Tais informações são muito importantes para a análise crítica das medidas de controle a serem tomadas. Em vista disso, o objetivo do presente estudo foi avaliar a qualidade microbiológica e a temperatura de dietas enterais, a fim de implantar o sistema APPCC na Central de Alimentação Enteral (CAE) do Hospital de Clínicas de Porto Alegre (HCPA).

\section{M É T O D O S}

Durante o período de planejamento de implantação do APPCC (setembro de 2001 a janeiro de 2002), a rotina diária da CAE do HCPA foi acompanhada desde a aquisição de matéria-prima até o preparo e a distribuição aos pacientes. Cada etapa foi observada e analisada criticamente, sendo seus perigos identificados. $\mathrm{O}$ resultado das análises microbiológicas também foi considerado para a identificação dos perigos nessas etapas. Para a elaboração e implantação do plano APPCC, os Pontos Críticos de Controle (PCC) foram identificados, assim como seus respectivos limites críticos e de segurança, procedimentos de monitorização, ações corretivas e procedimentos de verificação. Foram implantadas medidas preventivas para cada PCC. Nesse período, também foram realizados treinamentos com todas as pessoas envolvidas nas atividades desse setor, a fim de implementar as medidas preventivas. Os PCC foram definidos a partir de diagramas decisórios extraídos e adaptados da Portaria 46, de 10/02/1998, do Ministério da Agricultura e Abastecimento ${ }^{7}$.

Durante o período de implantação do sistema APPCC na CAE do HCPA foram coletadas amostras dos 4 tipos de dietas enterais produzidas: dietas I, II, III e dieta especial. A dieta I consistia de uma fórmula polimérica em pó a $22 \%$, apresentando em 100mL, 3,97g de proteínas; 3,36g de lipídios e 13,61 g de carboidratos. A dieta II era uma fórmula polimérica em pó a $27 \%$ com $4,88 \mathrm{~g}$ de proteínas; $4,12 \mathrm{~g}$ de lipídios e $16,7 \mathrm{~g}$ de carboidratos em $100 \mathrm{~mL}$. Já a dieta III era uma fórmula polimérica em pó a $34,5 \%$, sendo encontrado em $100 \mathrm{~mL}, 6,22 \mathrm{~g}$ de proteínas; $5,27 \mathrm{~g}$ de lipídios e $21,33 \mathrm{~g}$ de carboidratos. A dieta Especial era uma dieta em pó, polimérica ou oligomérica apresentando diferentes composições nos nutrientes, conforme indicação específica. Semanalmente, 
amostras de $80 \mathrm{ml}$ foram coletadas de cada uma dessas dietas por 20 semanas, em dois pontos do processo: a) logo após o preparo e b) após um período de armazenamento de 16 horas, sob refrigeração, na Copa Sul do $6^{\circ}$ andar do HCPA, por esta copa apresentar média de temperatura elevada em relação às demais que atendem pacientes do Sistema Único de Saúde (SUS). Ao todo, foram coletadas 320 amostras, sendo 160 antes e 160 após a implementação do sistema APPCC.

Todas as amostras coletadas foram submetidas a análises microbiológicas em laboratório terceirizado e consistiram na contagem de microorganismos mesófilos totais, na determinação do número mais provável de coliformes totais e coliformes fecais pela técnica dos tubos múltiplos, contagens de Staphylococcus aureus, Bacillus cereus, Clostridium sulfito redutores e presença de Salmonella sp. As análises foram realizadas de acordo com os métodos preconizados pela American Public Health Association ${ }^{8}$. Também foram quantificados os microorganismos Yersinia enterocolitica por método proposto por Schiermann ${ }^{9}$ e Listeria monocytogenes, segundo método descrito por Food and Drug Administration?.

O liquidificador e a peneira utilizados na preparação das formulações enterais foram analisados microbiologicamente a partir da coleta de amostras utilizando suabes estéreis. Foram realizadas duas coletas e os suabes foram passados em diversos pontos do liquidificador e na peneira, sendo em seguida colocados em água peptonada $0,1 \%$ para a pesquisa dos microorganismos já descritos acima.

Para este estudo foram mensuradas as temperaturas dos refrigeradores onde foram armazenadas as dietas desde novembro de 2001 a março de 2002, todos os dias nos seguintes horários: 7h, 10h, 13h, 16h e 19h, sendo realizada a média mensal das temperaturas observadas nas copas $3^{\circ} \mathrm{Sul}$ e $4^{\circ} \mathrm{Sul}$ (únicas que atendem convênios e particulares) e nas demais copas do HCPA que atendem pacientes do SUS.
Os dados das amostras foram analisados no programa SPSS (Versão 12.0, Inc, Chicago). Foram utilizadas médias e desvio-padrão para descrever os resultados das análises de microorganismos mesófilos totais, coliformes fecais e totais de cada dieta analisada. Para comparação dos dados coletados antes e após a implantação do sistema APPCC foi utilizado o teste de Wilcoxon.

\section{RESULTADOSE DISCUSSÃO}

Pela análise dos perigos foram identificadas etapas do processo no qual contaminações microbiológicas poderiam ser controladas, porém por medidas diferentes das Boas Práticas de Fabricação (BPF), sendo, portanto, classificadas como PCC. As etapas que eram controladas pelas BPF foram consideradas Pontos de Controle (PC). A produção das dietas enterais no HCPA consiste em: 1) Aquisição de matéria-prima (PCC); 2) Recepção das dietas; 3) Armazenamento; 4) Pesagem de produtos nutricionais em pó (PC); 5) Incorporação da água e homogeneização da mistura $(P C)$; 6) Envase e rotulagem; 7) Conferência; 8) Armazenamento (PCC); 9) Distribuição às unidades; 10) Reaquecimento e 11) Distribuição ao paciente;

A aquisição da matéria-prima foi considerada um PCC, uma vez que os produtos nutricionais podem estar contaminados por microorganismos patogênicos em níveis inaceitáveis e, mesmo aplicando medidas adequadas de higiene e manipulação, tais perigos não são reduzidos até níveis aceitáveis. Além disso, o fato de não existir um processamento subseqüente capaz de eliminar ou reduzir essa contaminação contribui para que essa etapa seja avaliada como PCC. Nessa etapa, a medida preventiva identificada foi a exigência de laudos de análises microbiológicas no momento da aquisição dos produtos.

Antes da implantação do APPCC, cada fornecedor emitia laudos relativos à qualidade da matéria-prima no momento da aquisição do produto, segundo critérios de qualidade próprios. Com 
a implantação do APPCC foi estabelecido que todos os laudos deveriam seguir um mesmo padrão, conforme a Resolução - RDC no 12, de 2 de janeiro de 2001 - da Agência Nacional de Vigilância Sanitária, a qual define critérios e padrões microbiológicos para alimentos ${ }^{10}$.

Nas etapas de recepção e armazenamento dos produtos não foram identificados perigos de contaminação microbiológica, uma vez que os produtos são considerados estáveis (pó) e armazenados nas próprias embalagens.

As etapas de pesagem dos produtos em pó e homogeneização das misturas foram consideradas pontos de controle (PC), uma vez que os perigos identificados poderiam ser controlados pelos procedimentos descritos no Manual de Boas Práticas de Fabricação da CAE do HCPA. Os riscos de contaminação por manipulação ou por higienização inadequadas dos utensílios utilizados nesses procedimentos foram considerados bem controlados, não havendo, portanto, necessidade de alterações nessas etapas, o que é confirmado pelas análises microbiológicas realizadas no liquidificador e na peneira usados na homogeneização, que não evidenciaram qualquer contaminação.

Anteriormente à implantação do APPCC, o produto homogeneizado era colocado em jarras plásticas higienizadas e armazenado em refrigerador por 30 minutos, visando diminuir a espuma antes do envase. Após a implantação do APPCC, essa etapa foi eliminada para diminuir a manipulação e a possibilidade de contaminação. Para isso, a mistura passou a ser envasada em frascos descartáveis, utilizando balança digital para garantir correto fracionamento e precisão do volume prescrito ao paciente.

Também, como conseqüência da implantação do sistema, os frascos contendo as dietas enterais, já rotulados e conferidos por uma técnica de nutrição, passaram a ser armazenados sob refrigeração não mais nas copas, mas sim na própria $\mathrm{CAE}$, até a distribuição às unidades de internação. A mudança do local de armazenamento ocorreu em decorrência dos resultados das medidas de temperatura dos refrigeradores das copas, dos quais, $92 \%$ apresentaram médias de temperaturas consideradas inadequadas (acima de $5^{\circ} \mathrm{C}$ - Tabela 1). Embora a temperatura de $7^{\circ} \mathrm{C}$ seja suficiente para inibir o crescimento de diversos patógenos ou deteriorantes alimentares ${ }^{11}$, a legislação brasileira atual exige temperaturas de refrigeração abaixo de $5^{\circ} \mathrm{C}^{12}$. Pelos resultados apresentados neste estudo os refrigeradores foram substituídos, visando garantir a qualidade do alimento armazenado.

Tabela 1. Média das temperaturas do refrigerador de armazenamento das dietas enterais do HCPA antes da implantação do sistema APPCC nos período de novembro de 2001 a março de 2002. Porto Alegre.

\begin{tabular}{|c|c|c|c|c|c|c|}
\hline \multirow{2}{*}{ Copas } & \multicolumn{5}{|c|}{ Média de temperatura dos refrigeradores das copas $\left({ }^{\circ} \mathrm{C}\right)$} & \multirow{2}{*}{ Temperatura média } \\
\hline & Novembro/2001 & Dezembro/2001 & Janeiro/2002 & Fevereiro/2002 & Março/2002 & \\
\hline $3^{\circ} \mathrm{Sul}$ & 10,1 & 12,0 & 13,6 & 11,8 & 14,0 & 12,30 \\
\hline $4^{\circ} \mathrm{Sul}$ & 4,6 & 4,2 & 4,6 & 6,4 & 9,0 & 5,76 \\
\hline $4^{\circ}$ Norte & 9,3 & 9,9 & 5,0 & 3,6 & 5,1 & 6,58 \\
\hline $5^{\circ}$ Norte & 11,2 & 7,0 & 5,9 & 14,7 & 13,1 & 10,38 \\
\hline $5^{\circ} \mathrm{Sul}$ & 11,8 & 11,6 & 11,5 & 7,3 & 12,3 & 10,90 \\
\hline $6^{\circ} \mathrm{Sul}$ & 10,8 & 11,9 & 12,7 & 11,6 & 13,1 & 12,02 \\
\hline $7^{\circ} \mathrm{Sul}$ & 11,0 & 7,8 & 11,6 & 9,3 & 9,4 & 9,82 \\
\hline $7^{\circ}$ Norte & 4,6 & 7,2 & 6,5 & 6,4 & 7,1 & 6,36 \\
\hline $8^{\circ} \mathrm{Sul}$ & 2,8 & 7,8 & 9,4 & 8,0 & 9,3 & 7,46 \\
\hline $8^{\circ}$ Norte & 8,8 & 7,7 & 8,6 & 8,5 & 11,5 & 9,02 \\
\hline $9^{\circ}$ Norte & 4,3 & 6,2 & 5,4 & 3,6 & 4,2 & 4,74 \\
\hline $13^{\circ}$ Norte & 6,9 & 5,7 & 5,4 & 5,9 & 5,2 & 5,82 \\
\hline
\end{tabular}

HCPA: Hospital de Clínicas de Porto Alegre; APPCC: Análise de Perigos e Pontos Críticos de Controle. 
À etapa de armazenamento das fórmulas preparadas foi considerada o segundo PCC do processo, pois há risco de ocorrer a multiplicação de microorganismos presentes na preparação. Mesmo que as análises microbiológicas tenham demonstrado ausência ou baixas contagens de microorganismos patogênicos nas amostras das dietas enterais, a possibilidade da presença de patógenos ainda existe. Nesse caso, havendo abuso de tempo e temperatura na etapa de armazenamento, a proliferação microbiana pode ocorrer a ponto de comprometer de forma significativa a saúde dos pacientes ou a qualidade final do produto. Outro fator que define essa etapa como um PCC é a existência de medida preventiva (controle de temperatura), que pode ser tomada para reduzir os perigos até níveis aceitáveis e a não existência de uma etapa subseqüente que elimine ou reduza esses perigos.

Durante as etapas de distribuição às unidades, reaquecimento e distribuição das dietas aos pacientes não foi identificada a possibilidade de perigos biológicos, uma vez que o tempo de distribuição aos pacientes é bastante pequeno (máximo de 30 minutos), e isso dificulta a multiplicação de microorganismos até doses infectantes perigosas ou mesmo liberação de toxinas.

Foram coletadas 320 amostras, sendo 160 antes e 160 após implementação do APPCC. Na Tabela 2 estão demonstradas as médias das contagens de microrganismos mesófilos totais presentes nas dietas após preparo e nas dietas armazenadas (16h), antes e após a implantação do APPCC. Comparando-se as dietas antes e após APPCC, foram encontradas reduções estatisticamente significantes para as contagens absolutas de algumas amostras. As médias das contagens de mesófilos totais das dietas padrão II, III e dieta especial, antes do APPCC, eram de 0,71; 0,69 e $2,68 \mathrm{UFC} / \mathrm{mL} \times 10^{2}$, respectivamente. Essas contagens reduziram-se significantemente para 0 UFC $/ \mathrm{mL} \times 10^{2}$ nas dietas II, III e Especial após APPCC. Em relação à dieta I não houve diferença estatisticamente significante antes e após APPCC em ambos momentos da coleta das amostras.

Tabela 2. Comparação das médias das contagens de microorganismos mesófilos totais presentes nas dietas (após preparo e após 16h de armazenamento), antes e após a implantação do APPCC no Hospital de Clínicas de Porto Alegre, 2002.

\begin{tabular}{|c|c|c|c|}
\hline Dieta após preparo & Médias & Desvios-padrão (UFC/mL) & Valor $p$ \\
\hline I - antes APPCC & $0,325 \times 10^{2}$ & $\left(0,64 \times 10^{2}\right)$ & \multirow{2}{*}{0,208} \\
\hline I - após APPCC & $0,4 \times 10^{2}$ & $\left(1,74 \times 10^{2}\right)$ & \\
\hline II - antes APPCC & $0,71 \times 10^{2}$ & $\left(1,86 \times 10^{2}\right)^{\star}$ & \multirow{2}{*}{0,018} \\
\hline II - após APPCC & ND & ND & \\
\hline III - antes APPCC & $0,69 \times 10^{2}$ & $\left(2,02 \times 10^{2}\right)^{*}$ & \multirow{2}{*}{0,026} \\
\hline III - após APPCC & ND & ND & \\
\hline Especial - antes APPCC & $2,68 \times 10^{2}$ & $\left(9,15 \times 10^{2}\right)$ * & \multirow{2}{*}{0,028} \\
\hline Especial - após APPCC & ND & ND & \\
\hline \multicolumn{4}{|l|}{ Dieta Armazenada (16h) } \\
\hline I - antes APPCC & $3,55 \times 10^{2}$ & $\left(7,66 \times 10^{2}\right)$ & \multirow{2}{*}{0,17} \\
\hline I - após APPCC & $4,83 \times 10^{2}$ & $\left(12,13 \times 10^{2}\right)$ & \\
\hline II - antes APPCC & $92,91 \times 10^{2}$ & $\left(401,89 \times 10^{2}\right)^{*}$ & \multirow{2}{*}{0,047} \\
\hline$\|$ - após APPCC & $1,64 \times 10^{2}$ & $\left(6,92 \times 10^{2}\right)$ & \\
\hline III - antes APPCC & $1,15 \times 10^{2}$ & $\left(1,72 \times 10^{2}\right)^{\star}$ & \multirow{2}{*}{0,005} \\
\hline III - após APPCC & ND & & \\
\hline Especial - antes APPCC & $3,25 \times 10^{2}$ & $\left(12,92 \times 10^{2}\right)$ & \multirow{2}{*}{0,116} \\
\hline Especial - após APPCC & $0,14 \times 10^{2}$ & $\left(0,43 \times 10^{2}\right)$ & \\
\hline
\end{tabular}

UFC/mL: Contagem de microorganismos por mL; APPCC: Análise de Perigos e Pontos Críticos de Controle; I: Dieta I; II: Dieta II; III: Dieta III; Especial: Dieta especial; ND: não detectado; $p$ : resultado do teste estatístico; *significância estatística, valor $p$ significante: $<0,05$. 
Na comparação de dietas após a refrigeração, reduziu-se significantemente de 92,91 UFC/mL x 102 para 1,64 UFC/mL x 102 na dieta II após APPCC. Também reduziu-se de 1,15 para 0 UFC/mL x 102 na dieta III após APPCC. Nessas dietas refrigeradas, a implantação do APPCC também demonstrou resultados ao haver diminuição significante da média de mesófilos das dietas padrão II e III.

De acordo com a Resolução RCD n 63, de 6 de julho de 2000'13, a contagem de coliformes totais presentes em dietas enterais deve ser menor que $3 \mathrm{UFC/g}$ e de mesófilos totais deve ser menor que $103 \mathrm{UFC/g}$. Em relação às análises de coliformes totais, 1 amostra (1,25\%) estava contaminada nas dietas prontas (após preparo) e 9 $(11,25 \%)$ também estavam acima dos padrões de contagem de coliformes na etapa após $16 \mathrm{~h}$ de refrigeração, ambas antes do APPCC (Tabela 3).
Depois da implantação do APPCC, o percentual de contaminação, praticamente, manteve-se o mesmo, verificando-se contaminação por coliformes totais em uma amostra $(1,25 \%)$ das dietas prontas e em oito amostras (10,00\%) após refrigeração. Em relação às análises de coliformes totais nas dietas, não houve nenhuma diferença estatisticamente significante entre as dietas analisadas antes ou depois da implantação do APPCC, demonstrando que as boas práticas já existiam antes do APPCC, mas que necessitam sempre ser reforçadas por treinamentos sistemáticos com todos os funcionários, para corrigir os determinantes dessa contaminação.

Das 80 amostras analisadas na etapa após preparo, antes do APPCC, 13 (16,25\%) estavam com níveis de microorganismos mesófilos totais acima do permitido segundo parâmetros estipulados pela RDC $n^{\circ} 63$. Na etapa após $16 \mathrm{~h}$ de

Tabela 3. Análises microbiológicas de coliformes totais (UFC/mL $\times 10^{2}$ ) realizadas antes e depois da implantação do Sistema APPCC na Central de Alimentos Enterais do HCPA. Alimentação Enteral do Hospital de Clínicas de Porto Alegre, de setembro de 2001 a janeiro de 2002.

\begin{tabular}{|c|c|c|c|c|c|c|c|c|c|c|c|c|c|c|c|c|}
\hline \multirow{3}{*}{ Semana } & \multicolumn{8}{|c|}{ Antes da implantação do APPCC } & \multicolumn{8}{|c|}{ Depois da implantação do APPCC } \\
\hline & \multicolumn{4}{|c|}{ Pós preparo } & \multicolumn{4}{|c|}{ Pós refrigeração } & \multicolumn{4}{|c|}{ Pós preparo } & \multicolumn{4}{|c|}{ Pós refrigeração } \\
\hline & I & $\|$ & III & $E$ & I & $\|$ & III & $E$ & । & $\|$ & III & $\mathrm{E}$ & । & $\|$ & III & $E$ \\
\hline 1 & - & - & - & - & - & - & - & - & - & 2,8 & - & - & 40 & - & - & - \\
\hline 2 & - & - & - & - & - & - & - & - & - & - & - & - & 35 & - & - & - \\
\hline 3 & - & - & - & - & - & - & - & - & - & - & - & - & - & - & - & - \\
\hline 4 & - & - & - & - & - & - & - & - & - & - & - & - & 0,6 & - & - & - \\
\hline 5 & - & - & - & - & - & - & - & - & - & - & - & - & - & - & - & - \\
\hline 6 & - & - & - & - & 1,2 & 1,1 & 1,8 & - & - & - & - & - & - & - & - & - \\
\hline 7 & - & - & - & - & - & 1.800 & 1,8 & - & - & - & - & - & - & - & - & - \\
\hline 8 & - & - & - & 1,8 & - & - & - & - & - & - & - & - & - & - & - & - \\
\hline 9 & - & - & - & - & - & - & - & - & - & - & - & - & - & - & - & 1,6 \\
\hline 10 & - & - & - & - & - & - & - & - & - & - & - & - & - & - & - & 1,2 \\
\hline 11 & - & - & - & - & - & - & - & - & - & - & - & - & - & 1,8 & - & - \\
\hline 12 & - & - & - & - & - & - & - & - & - & - & - & - & - & - & - & - \\
\hline 13 & - & - & - & - & - & - & - & - & - & - & - & - & - & 3,1 & - & - \\
\hline 14 & - & - & - & - & - & - & - & - & - & - & - & - & 2,1 & - & - & - \\
\hline 15 & - & - & - & - & - & - & - & - & - & - & - & - & - & - & - & - \\
\hline 16 & - & - & - & - & 2,1 & 1,8 & 1,2 & 2,1 & - & - & - & - & - & - & - & - \\
\hline 17 & - & - & - & - & - & - & - & - & - & - & - & - & - & - & - & - \\
\hline 18 & - & - & - & - & - & - & - & - & - & - & - & - & - & - & - & - \\
\hline 19 & - & - & - & - & - & - & - & - & - & - & - & - & - & - & - & - \\
\hline 20 & - & - & - & - & - & - & - & - & - & - & - & - & - & - & - & - \\
\hline
\end{tabular}

Dieta enteral padrão= I, II, III e dieta enteral especial= E; Medidas não descritas = ausência $\left(0,0 \times 10^{2}\right.$ UFC/mL) de coliformes totais; I: Dieta I; II: Dieta II; III: Dieta III; Especial: Dieta especial; APPCC: Análises de Perigos e Pontos Críticos de Controle; HCPA: Hospital de Clínicas de Porto Alegre. 
refrigeração, antes do APPCC, 35 das 80 amostras $(43,75 \%)$ também haviam ultrapassado esse limite (Tabela 4). Depois da implantação do APPCC, das 80 amostras analisadas das dietas após preparo, apenas $1(1,25 \%)$ estava com níveis acima do permitido para microorganismos mesófilos aeróbios, enquanto 7 amostras (8,75\%) analisadas após refrigeração demonstraram níveis superiores desses microorganismos. Nenhum microorganismo patogênico como, Staphylococcus aureus, Bacillus cereus, Clostridium sulfito redutores e Salmonella sp. foram encontrados nas 320 amostras analisadas, seja antes ou após a implantação do APPCC. Isso demonstra a importância do seguimento às boas práticas, instituídas anteriormente na CAE.

No Anexo está o resumo do plano APPCC para dietas enterais produzidas no HCPA ${ }^{16-18}$.

Santos \& Tondo ${ }^{14}$ analisaram 25 amostras de fórmulas enterais manipuladas no lactário do
HCPA e demonstraram que 11 (44\%) delas estavam acima do padrão estabelecido para contagem total de mesófilos totais, enquanto que 6 (24\%) estavam acima dos limites estabelecidos para coliformes, segundo a RDC n ${ }^{\circ} 63$. Assim como o estudo de Lima et al. ${ }^{15}$, segundo o qual do total de amostras analisadas, $25 \%$ apresentaram contaminação por coliformes totais em dietas enterais, não indicando, necessariamente, contaminação fecal ou presença de patógenos; porém a presença de um número elevado desses microorganismos sugere condições sanitárias insatisfatórias.

Oliveira et al. ${ }^{1}$ analisaram amostras de alimentos enterais quanto à qualidade microbiológica e à temperatura antes e depois da implementação do APPCC. A contagem média de bactérias foi reduzida de $105 \mathrm{UFC} / \mathrm{mL}$ para < $101 \mathrm{UFC} / \mathrm{mL}$, após implantação desse sistema. Patchell et al. ${ }^{5}$ demonstraram que treinamento e implementação

Tabela 4. Análises microbiológicas de mesófilos totais (UFC/mL x 102) realizadas antes e depois da implantação do Sistema APPCC na Central de Alimentos Enterais do HCPA. Hospital de Clínicas de Porto Alegre, de setembro de 2001 a janeiro de 2002.

\begin{tabular}{|c|c|c|c|c|c|c|c|c|c|c|c|c|c|c|c|c|}
\hline \multirow{3}{*}{ Semana } & \multicolumn{8}{|c|}{ Antes da implantação do APPCC } & \multicolumn{8}{|c|}{ Depois da implantação do APPCC } \\
\hline & \multicolumn{4}{|c|}{ Pós preparo } & \multicolumn{4}{|c|}{ Pós refrigeração } & \multicolumn{4}{|c|}{ Pós preparo } & \multicolumn{4}{|c|}{ Pós refrigeração } \\
\hline & 1 & $\|$ & III & $\mathrm{E}$ & I & $\|$ & III & $\mathrm{E}$ & I & $\|$ & III & $\mathrm{E}$ & I & $\|$ & III & $\mathrm{E}$ \\
\hline 1 & - & - & - & - & - & - & - & - & 0,2 & - & - & - & 40 & - & - & - \\
\hline 2 & - & 0,4 & - & 41 & - & - & - & - & - & - & - & - & 35 & - & - & - \\
\hline 3 & - & - & - & - & 6,0 & 2,8 & - & - & - & - & - & - & - & - & - & - \\
\hline 4 & - & - & - & - & - & - & - & - & - & - & - & - & 0,6 & - & - & - \\
\hline 5 & - & - & 0,6 & 1,2 & 1,8 & 1,7 & 0,9 & 4,4 & - & - & - & - & - & - & - & - \\
\hline 6 & 2,3 & - & - & - & 1,2 & 2,9 & 2,6 & - & - & - & - & - & - & - & - & - \\
\hline 7 & - & - & - & - & - & 1.800 & 1,8 & - & - & - & - & - & - & - & - & - \\
\hline 8 & - & - & 2,0 & 1,8 & 2,1 & - & - & - & - & - & - & - & - & - & - & - \\
\hline 9 & - & - & - & 2,1 & 35 & 39 & 1,8 & 58 & - & - & - & - & - & - & - & 1,6 \\
\hline 10 & - & - & - & - & 5,1 & - & 3,1 & - & - & - & - & - & - & - & - & 1,2 \\
\hline 11 & 0,5 & - & - & - & 1,0 & 1,8 & 0,8 & - & - & - & - & - & - & 1,8 & - & - \\
\hline 12 & - & - & 0,6 & 0,7 & - & - & - & - & - & - & - & - & - & - & - & - \\
\hline 13 & - & 0,3 & - & - & 4,0 & 4,1 & 3,2 & - & - & - & - & - & - & 31 & - & - \\
\hline 14 & 0,8 & - & - & - & 1,0 & 1,2 & 0,8 & 0,2 & - & - & - & - & 21 & - & - & - \\
\hline 15 & - & 8,2 & - & - & 2,8 & 1,1 & 6,8 & 0,4 & - & - & - & - & - & - & - & - \\
\hline 16 & - & - & - & - & 2,1 & 1,8 & 1,2 & 2,1 & 7,8 & - & - & - & - & - & - & - \\
\hline 17 & - & 0,9 & - & - & 1,2 & - & - & - & - & - & - & - & - & - & - & - \\
\hline 18 & 0,7 & 0,6 & 0,6 & - & 1,4 & 1,9 & - & - & - & - & - & - & - & - & - & - \\
\hline 19 & 1,8 & 2,0 & 9,0 & - & - & - & - & - & - & - & - & - & - & - & - & - \\
\hline 20 & 0,4 & 1,8 & 1,1 & 6,9 & 6,3 & - & - & - & - & - & - & - & - & - & - & - \\
\hline
\end{tabular}

Dieta enteral padrão: I, II, III e dieta enteral especial: E; Medidas não descritas: ausência (0,0 x 102 UFC/mL) de mesófilos totais; I: Dieta I; II: Dieta II; III: Dieta III; Especial: Dieta especial; APPCC: Análise de Perigos e Pontos Críticos de Controle; HCPA: Hospital de Clínicas de Porto Alegre. 
de práticas de higiene, com a modificação do protocolo de alimentação enteral, reduziram a contaminação microbiológica das dietas de $62 \%(n=29)$ para apenas $6 \%(n=36)$ em pacientes que recebiam alimentação domiciliar, e de $45 \% \quad(n=62)$ para $4 \%(n=77)$ em pacientes recebendo dieta no hospital 5 .

\section{O N C L U S Ã O}

Com base nos resultados apresentados, a implantação do sistema APPCC contribuiu para o controle dos procedimentos executados na CAE, além de favorecer a diminuição efetiva das contagens microbianas das dietas ali produzidas.

A implantação desta metodologia também possibilitou medidas para a melhoria de equipamentos e para a padronização dos processos, favorecendo a garantia da qualidade do produto final.

\section{REFERÊ N CIAS}

1. Oliveira MR, Batista CR, Aidoo KE. Application of hazard analysis critical control points system to enteral tube feeding in hospital. J Hum Nutr Diet. 2001; 14(5):397-403.

2. Ribeiro NF. Análise de perigos e pontos críticos de controle na unidade de alimentação e nutrição (UAN). In: Fernandes AT. Infecção hospitalar e suas interfaces na área da saúde. São Paulo: Atheneu; 2000. v.2, capítulo 66, p.1247-55.

3. Okuma T, Nakamura M, Totake H, Fukunaga Y. Microbial contamination of enteral feeding formulas and diarrhea. Nutrition. 2000; 16(9): 719-22.

4. Oliveira GPC, Waitzberg DL. Contaminação microbiológica em nutrição enteral. In: Waitzberg DL. Nutrição Enteral e Parenteral na Prática Clínica. 3a.ed. São Paulo: Atheneu; 2001. Capítulo 40, p.649.

5. Patchell CJ, Anderton A, Holden C, MacDonald A, George RH, Booth IW. Reducting bacterial contamination of enteral feeds. Arch Dis Child. 1998; 78(2):166-8.

6. Silva EAJ. Manual de controle higiênico-sanitário em alimentos. 4a.ed. São Paulo: Livraria Varela; 2001.
7. Brasil. Portaria n.46 de 10 de fevereiro de 1998 Manual genérico de procedimentos para APPCC em indústrias de produtos de origem animal. Ministério da Agricultura e do Abastecimento. Diário Oficial da União. 199816 mar; Seção 1.

8. Speck ML. Compendium of methods for the microbiological examination of foods. 2nd ed. Washington (DC): American Public Health Association; 1984

9. Food and Drug Administration. Bacteriological manual. 7th ed. Rockville (MD); 1992.

10. Brasil. Agência Nacional de Vigilância Sanitária. Resolução RDC n.12, de 2 de janeiro de 2001. Regulamento técnico sobre padrões microbiológicos para alimentos [acesso em 2 jan 2001]. Disponível em: http://www.anvisa.gov.br

11. Jay JM. Parâmetros Intrínsecos e extrínsecos dos alimentos que afetam o crescimento microbiano. In: Jay JM. Microbiologia de alimentos. 6a.ed. Porto Alegre: Artmed; 2005. Capítulo 3, p.51-72

12. Brasil. Agência Nacional de Vigilância Sanitária. Resolução RDC n.216, de 15 de setembro de 2004. Regulamento técnico de boas práticas para serviços de alimentação. Diário Oficial da União. 200416 set; Seção 1.

13. Brasil. Agência Nacional de Vigilância Sanitária. Resolução RDC n.63, de 6 de julho de 2000. Regulamento técnico para terapia de nutrição enteral. Diário Oficial da União. 20007 jul.

14. Santos MISS, Tondo EC. Determinação de perigos e pontos críticos de controle para implantação de sistema de análise de perigos e pontos críticos de controle em lactário. Rev Nutr. 2000; 13(3): 211-22.

15. Lima ARC, Barros LM, Rosa MS, Cardonha MAS, Dantas MAM. Avaliação microbiológica de dietas enterais manipuladas em um hospital. Acta Cir Bras. 2005; 20(1):27-30.

16. Elementos de apoio para o sistema APPCC. 2a.ed. Brasília: SENAI; 2000. Serie Qualidade e Segurança Alimentar. Projeto APPCC Indústria. Convênio CNI/SENAI/SEBRAE.

17. Guia para elaboração do plano APPCC; laticínios e sorvetes. 2a. ed. Brasília: SENAl; 2000. Serie Qualidade e Segurança Alimentar. Projeto APPCC Indústria. Convênio CNI/SENAI/SEBRAE.

18. Guia de verificação do sistema APPCC. 2a.ed. Brasília: SENAI, 2000. Serie Qualidade e Segurança Alimentar. Projeto APPCC Indústria. Convênio CNI/SENAI/SEBRAE.

Recebido em: 22/2/2006

Versão final reapresentada em: 4/10/2006

Aprovado em: 21/11/2006 
148 | M.I.S.S. sIMON et al.

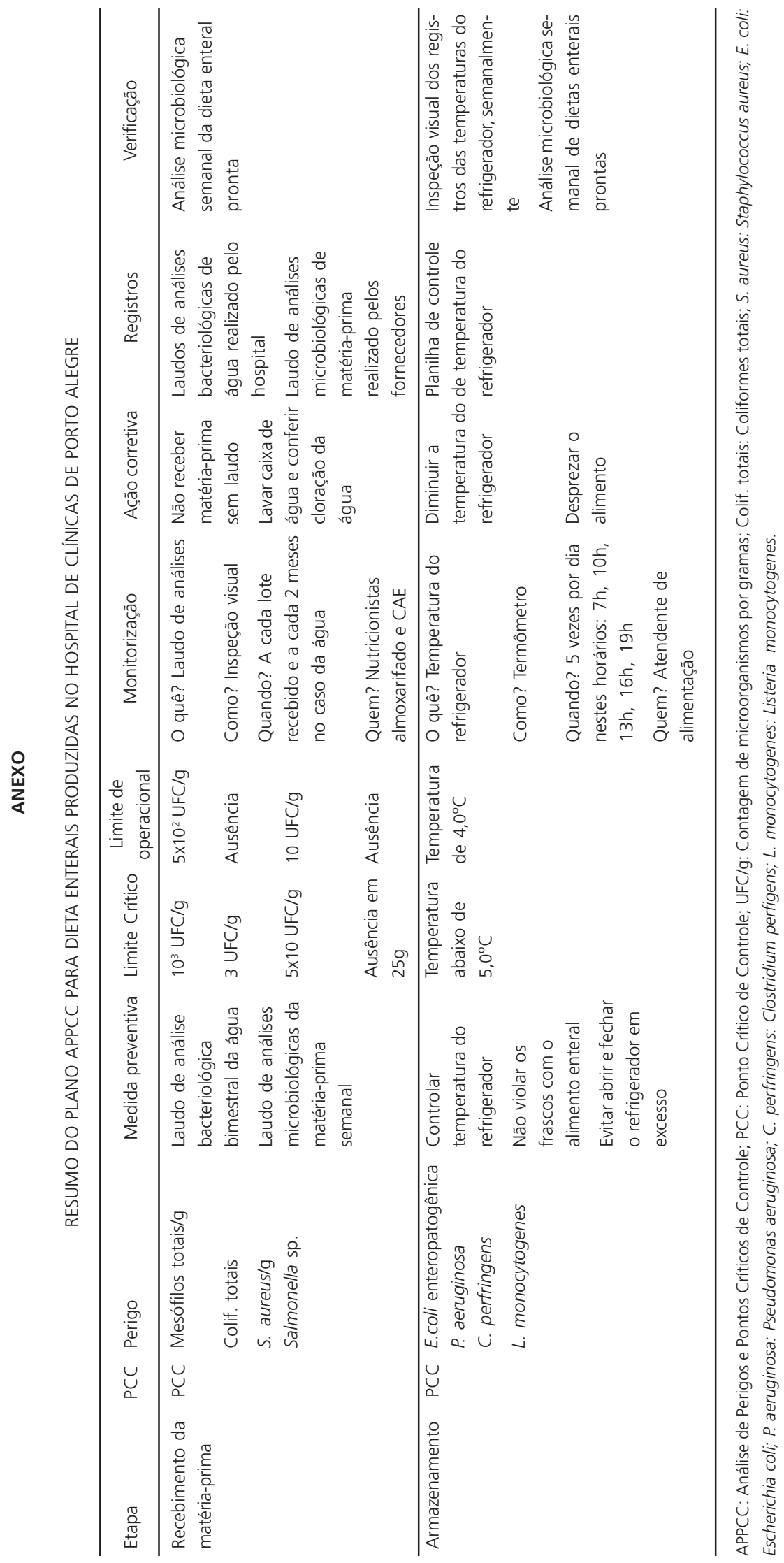

